\title{
Anxiety Construct in Higher Education
}

\section{Sudha Singh}

Asst. Prof. Dept. of Teacher Education, SHUATS, Naini, Allahabad, U.P. India

Corresponding author: sudhasinghno.1@gmail.com

\begin{abstract}
This paper is a review of journal articles, on general anxiety, from 2000 to 2016. It was found that more work had been done on the school population, than in higher education. Also, it was seen that in higher education the students selected, were mostly medical or engineering students. Other types of anxiety were explored in higher education, like academic anxiety etc.
\end{abstract}

Keywords: School population, higher education, students, academic

Anxiety has come to mean something that disturbs, is unpleasant and has undesired repercussions, in common parlance, or layman's language. This construct has pervaded the lives of all human beings, in one way or another. When it helps to remove laziness or sluggishness, then it is considered beneficial or positive anxiety. When it acts as an impediment and causes harm, it becomes a matter of concern. Further, when it exceeds the limit, it takes the form of a psychological disorder. A lot of work has been done on psychological disorders, but very little concern has been shown about the general anxiety levels of students, pursuing higher education. These students will be the future of the country, so, knowledge of their anxiety becomes very important.

Rollo May (Popova, 2013), a psychologist, felt that a person would have no anxiety, if there were no possibility whatever. Creating and actualizing one's possibilities, always involves negative as well as positive aspects, leading to anxiety. The Encyclopedia of Psychology (2000), gave an elaborate definition of anxiety, saying that it was an emotion characterized by heightened autonomic system activity, specifically activation of the sympathetic nervous system i.e. increased heart rate, blood pressure, respiration and muscle tone, subjective feelings of tensions and cognitions that involve apprehension and worry. It is distress or uneasiness caused by fear of danger or misfortune, earnest but tense desire, a state of apprehension and 
psychic tension, a threat to some value which the individual holds essential to his existence as a personality (Webster's College Dictionary, (c) 2010).

Many psychologists have also tried to define anxiety. The famous psychologist, Sigmund Freud (1923, 1924), described anxiety as 'something felt', an unpleasant affective state or condition. This state in patients with anxiety-neurosis, was characterized by "all that is characterized be the word 'nervousness', apprehension or anxious expectation and efferent discharge phenomenon (Freud, 1923,1924). Anxiety is an unpleasant emotional reaction to real or imagined dangers, accompanied by autonomic discharge and subjectively experienced, such as tension, fright or nervousness (Beck, 1972).

Mathison (1977) defined anxiety as a state that has association with feelings of uncertainly, helplessness and threat to the core of personality. Anxiety in general causes vague diffuse feelings of uneasiness, distress, inner tension and worrying in advance. According to Spielberger (1983), during the $20^{\text {th }}$ century, the concept of anxiety attracted much attention from psychologists, since that period was known as the 'age of anxiety'. He felt that, generally speaking, if an individual perceives and appraises a particular situation as threatening, an unpleasant emotional reaction usually occurs. Gray and McNaughton (1996), defined anxiety as a state of the conceptual or central nervous system characterized by activity of the behavioral inhibition system (BIS). Almost all agree that 'the characteristics of anxiety as an emotion are that it is distressing and that its sources arc indefinite', (The Oxford Companion to the Mind, 2006).

In retrospect then, we can see that anxiety, when it is low or high, requires intervention, as it is bound to effect the student both physically and mentally, leading very often, to disastrous effects on academic output. For this purpose, a review of Indian researches on anxiety in higher education and tools for the purpose, revealed a lacunae, most surprising. Researchers have been done on types of anxiety, academic anxiety (Bhansali \& Trivets, 2008), English language anxiety (Hemamalini. 201 I), mathematics anxiety (Mahmood \& Khatoon, 2011), computer anxiety (Kanan et al. 2012), death anxiety (Latha et al. 2013) and so on. The study of anxiety with other variables like academic achievement (Mokashi, 2007) social acceptance, optimism, depression $\mathcal{E}$ stress (Kumaraswamy, 2013), mental health (Malhotra and Sabharwal, 2013), religious attitudes \& death attitudes (Laltha et al. 2013) etc., but most of them are on school children and not higher education. Also, they explore anxiety interventions and its relation with other variables.

From 2000 to 2016, an overview of research work done in India, on general anxiety, in higher education, was found to be an area, not much researched. Ganguli (2000) made an exploratory study to access depression, anxiety and stress among fresh medical students of Gujarat. Dwivedi and Gunthey (2005), investigated the effect of medium of instruction on academic anxiety of school students from Jodhpur city. Vazalwar and Yadav (2005), investigated the 
relationship of reading comprehension $\mathrm{n}$ English, with respect to anxiety, socio-economic status and school environment, of Bilaspur and Janjgir-Champa district of Chattisgarh state Mokashi (2007), identified the relationship between anxiety and scholastic achievement of residential school students conducted on two residential schools in Dharwad. Bhansali and Trivets (2008) aimed to find out the incidences and intensity of academic anxiety among adolescents. Ganihar and Wajiha (2009), examined the factors affecting academic achievement of IX standard students in Mathematics. Ghaderi et al. (2009), conduced a study to understand and compare the experiences of Stress, Anxiety, and depression among the Indian and the Iranian students. The data was collected from students studying in different departments of University of Mysore. Deb et al. (2010), researched to understand better, anxiety among adolescents in Kolkata city.

Sahoo and Khess (2010). studied the lifetime prevalence of depression, anxiety, and stress among adolescents and young adults around the world and found that it is currently estimated to range from $5 \%$ to $70 \%$, with an Indian study reporting no depression among college going adolescents. Hemamalini, (2011), made an attempt to find the relationship between English Language Anxiety and the English Achievement of High School Students of Ramanagaram city. Mahmood and Khatoon (2011), developed and validated a mathematics Anxiety Scale for Secondary and Senior secondary School Students from six secondary schools of Aligarh. Narula et al. (2011) explored the effectiveness of humour when used as intervention in large group teaching over negative emotions amongst students, on medical students of $4^{1}$ Semester of RMCH, Bareilly Singravelu (2011) developed a new instrument to measure students' mathematics anxiety, from VIII standard students of Pondicherry region. Vazalwar, (2011), explored the role of anxiety in L2 reading, on a sample of 960 standard XI students from 32 Senior Secondary Schools, of Bilaspur and Janjgir Champa districts of Chhattisgarh, Kannan et al. (2012), studied Computer Anxiety in Higher Secondary Students. Bakhla et al. (2013), studied anxiety in high in school going children.

Kumaraswamy (2013), described the research carried out in the last 3 decades especially regarding stress, anxiety \& depression, among college students. Latha et al. (2013), examined the relationship between death anxiety, life satisfaction and meaning, religiosity and selected personal factors among health care staff and students in three teaching hospitals - Kasturba Medical College (Manipal \& Mangalore). KVG. Medical College, Sullia and Dr. A.V. Baliga Hospital, Udirl Malhotra and Sabhanval (2013)-investigated the relation between anxiety and mental health of adolescents. Pant et al. (2013). examined yoga for controlling examination anxiety, depression and academic stress in students appearing for Indian Board Examination. Patel (2013), constructed and standardized an Anxiety Measurement Test for Students of Standard $11^{\text {th }}$ and $12^{\text {th }}$ of Anand District. 
(Y)

Singh and Jha (2013), assessed the level of anxiety and its relation with optimism and academic achievement among medical and engineering students Sridevi (2013), attempted to find out the relationship between test anxiety, general anxiety and academic achievement of higher secondary school students. Banga (2014), carried out a study, to find out that the academic anxiety among high school students in relation to different social categories, Mary et al. (2014) in their study analyzed the level of state anxiety among board exam attending school students in Tamil Nadu. Ahmed and Julius (2015),stated that there was a growing concern among educators about the low academic performance of college students and its influence on their mental health. Kumar, (2015), attempted to find out whether Yoga Intervention has any effect on Stale and Trait Anxiety and also on the Subjective well-being, in the Himalayan region. Roy et al. (2015) undertook to determine the presence of depression, anxiety and stress among first year medical undergraduates Bharati, (2016) investigated the anxiety level of sports and non-sports students during board exam.

\section{CONCLUSION}

In conclusion, therefore, we find that anxiety level in higher education is an area, that has been neglected. After Durganand Sinha, of Allahabad University, in the sixties, very few attempts were made to bridge this gap. The author developed an anxiety scale for undergraduate students (Singhal, 2016), to know the levels of anxiety prevailing in the higher education population in Allahabad. The anxiety of higher education students has an immediate effect on society and the country. They are the work force of our tomorrow. Therefore this is a very important sphere to be constantly investigated, to find any differences, that crop up with the rapidly changing times.

\section{REFERENCES}

Ahmed, Zarina and Julius, Sheela Hemlatha. 2015. 'Academic Performance, Resilience, Depression, Anxiety and Stress among Women College Students', Indian Journal of Positive Psychology, 6(4): 367-370.

Bakhla, A.K., Sinha, P., Sharan, R., Binay, Y., Verma, V. and Chaudhary, S. 2013. 'Anxiety in School Students: Role of Parenting and Gender', Indian Psychiatry Journal, 22: 131-7.

Banga, Chaman Lal, 2014. 'Academic Anxiety Among High School Students in Relation to Different Social Categories', International Multidisciplinary e-Journal, 3(3): 73-87.

Beck, A.T. 1972. 'The Phenomena of Depression: A Synthesis', in Offer and Freeman (1972), pp. 136-58.

Bhansali, Reena and Trivets, Kunjan. 2008. 'Is Academic Anxiety Gender Specific?: A Comparative Study', Journal of Social Sciences, New Delhi, 17(1): 1-3.

Bharati, Jaya. 2016. “Anxiety among U.P. Board Students in Uttar Pradesh, India', The international Journal of Indian Psychology, 3(2).

Deb, Sibnath, Chatterjee, Pooja and Walsh, Kerryann. 2010. "Anxiety Among High School Students in India: Comparisons Across Gender, School Type, Social Strata and Perceptions of Quality Time with Parents', Australian Journal of Educational and Developmental Psychology, 10: 18-31. 
Dwivedi, Neeta and Gunthey, Ravi. 2005. Influence of Medium of Instruction on Level of Academic Anxiety Among School Students, Edutracks, 5(4): 31-32.

Freud, S. (1923). The Ego and the Id'., S.E., 19: 12-66.

Freud, S. 1924. 'Infantile Organization of Libido: Supplement Theory Sexuality', Int. J. Psychoanalysis, 5: 125-129.

Ganguli, H.C. 2000. 'Epidemiologist Findings on Prevalence of Mental Disorders in India,' Indian Journal of Psychiatry, 42(1): 14-20.

Ganihar, N.N. and Wajiha, A.H. 2009. 'Factors Affecting Academic Achievement of IX Standard Students in Mathematics', Journal of Educational Studies, Allahabad, 6(1): 16-23.

Ghaderi, A.R., Venkatesli Kumar, G. and Sampath Kumar. 2009. ‘Depression, Anxiety and Stress among the Indian and Iranian Students', Journal of the Indian Academy of Applied Psychology, 35(1): 33-37.

Gray, J.A. and McNaughton, N. 1996. 'Comparison Between The Behavioural Effect of Sepal and Hippocampal Lesions: A Review', Neuroscience and Biobehavioral Reviews, 7: 119-88.

Hemamalini. H.C. 2011. 'Anxiety and Academic Achievement of High. School Students of Mysore City'. Journal of Community Guidance and Research, 28(1): 0970-B46..

Kaunas, B., Muthumanickam, A. and Chandrasekaran, S. 2012. 'Computer Anxiety among Higher Secondary Students', International Journal of Development Research, 2(4): 1008-1011.

Kumar, T. Shantha. 2015. 'How Anxiety and Depression can affect the Perceptual Process of Human Life? Exploring Human Values With Nature as a Secure Base and Focusing on Healthy life with Yoga and Meditation (An Empirical View of Himalayan Region)', The International Journal of Indian Psychology, 3(1).

Kumaraswamy, Narasappa. 2013. 'Academic Stress, Anxiety and Depression among College Students- A Brief Review', International Review of Soda! Sciences and Humanities, 5(1): 135-143.

Latha, K.S., Sahana, M., Mariella, D., Subbannayya, K. and Asha, K. 2013. 'Factors Related to Life satisfaction, Meaning of life. Religiosity and Death Anxiety in Health Care Staff and Students: A cross Sectional Study from India', Online J. Health Allied Scs., 12(2): 7.

Mahmood, Sadia and Khatoon, Taliira. 2011. 'Development and validation of the Mathematics Anxiety Scale for Secondary and Senior Secondary School Students', British Journal of Arts and Social Science, 2(2).

Malhotra, Taruna and Sabhanval, Slieena. 2013. 'Anxiety and Mental Health of Adolescents; A Study. International Educational E-Journal, (Quarterly), 2(3).

Mary, Revina Ann; Marslin, Gregory; Frankln, Gregory and Sheeba, Caroline J. 2014. Test Anxiety Levels of Board Exam Going Students in Tamil Nadu, India', Bio. Med. Research International, Vol. 2014, Article ID 578323, 9 pages.

Mathisou, Majorie, A. 1977. 'Curricular Interventions and Programming Innovations for the Reduction of Mathematics Anxiety', Paper presented at the Annual Convention of the American Psychological Association (San Francisco, California, August 26-30).

May, R. 1950. 'The Meaning of Anxiety', New York, Ronald Press.

Mokashi, Malavika V. 2007. 'Correlates of Anxiety and Scholastic Achievement of Residential Students', Thesis, Dept. of Human Development, College of Rural Home Science, Dharwad, Univ. of Agricultural Sciences, July, 2007.

Narula, Kamesh; Chaudhary, Varsha; Narula, Kiisum and Narayan, Ram. 2011. 'Depression, Anxiety and Stress Reduction in Medical Education: Humor as an Intervention'. Online Journal of Health and Allied Sciences, 10(1). 
$\mathcal{P}$ Singh

Pant, Gaurav; Bera, T.K. and Shefe, Sanjay Uddhav. 2013. 'Yoga For Controlling Examination Anxiety, Depression and Academic Stress among Students Appearing for Indian Board Examination', International Journal of Receni Scientific Research, 4(8): 1216- 1219.

Patel Mcliulkumar Kantilal. 2013. 'Construction and Standardization of Anxiety Measurement Test for Students of Standard $11^{\text {th }}$ and $12^{\text {th }}$ of Anand District'. Acme International Journal of Multidisciplinary Research, 1(2).

Roy Priya P., Sailesh, Kumar Sai and Doshi, M.A. 2015. 'Depression, Anxiety and Stress Among First Year Undergraduate Medical Students', Asian Journal of Biomedical and Pharmaceutical Sciences, 5(45): 37-38. 